\title{
Indústria 4.0 e Sociedade 5.0: visões comparadas
}

\author{
Industry 4.0 and Society 5.0: comparative views \\ Industria 4.0 y Sociedad 5.0: vistas comparativas
}

Recebido: 07/08/2021 | Revisado: 14/08/2021 | Aceito: 19/08/2021 | Publicado: 22/08/2021

\author{
Irapuan Glória Júnior \\ ORCID: https://orcid.org/0000-0003-2973-3470 \\ Universidade Paulista, Brasil \\ E-mail: ijunior@ndsgn.com.br \\ João Gilberto Mendes dos Reis \\ ORCID: https://orcid.org/0000-0001-6409-2299 \\ Universidade Paulista, Brasil \\ E-mail: betomendesreis@msn.com
}

\begin{abstract}
Resumo
As organizações têm sido apresentadas a uma nova forma de organização produtiva, a chamada de Indústria 4.0, com a promessa de integralização de seus processos, alta customização, alta produção e redução dos custos de produção. Baseado nos conceitos da Industria 4.0 surgiram diversas variações como a Logística 4.0, Saúde 4.0, e Agricultura 4.0. Porém, foi no Japão que surgiu a ideia Sociedade 5.0 que promete a aplicação de todos os aspectos da quarta revolução e seus desdobramentos em uma sociedade totalmente autônoma. O presente trabalho compara a Indústria 4.0 e a Sociedade 5.0 em relação a origem, elementos estruturantes e uso da tecnologia. A metodologia empregada foi uma pesquisa bibliográfica. $\mathrm{O}$ resultado apresenta uma lista de 10 características que comparam ano de criação do conceito, país de origem, necessidade motivadora, nível de urgência, foco, escopo de aplicação, emprego de tecnologia, grau de uso da inteligência artificial, elementos fundamentais e desdobramentos provenientes do conceito. As contribuições para a prática são do estabelecimento das diferenciações que podem contribuir para que estrategistas, investidores e gestores de produção possam compreender as diferenças e vislumbrarem um futuro próximo.
\end{abstract}

Palavras-chave: Indústria 4.0; Sociedade 5.0; Automação; Comparação; Tecnologia.

\begin{abstract}
Organizations have been introduced to a new form of productive organization, called Industry 4.0, with the promise of integrating their processes, high customization, high production and reduced production costs. Based on the concepts of Industry 4.0, several variations emerged, such as Logistics 4.0, Health 4.0, and Agriculture 4.0. However, it was in Japan that the idea Society 5.0 emerged, which promises the application of all aspects of the fourth revolution and its consequences in a fully autonomous society. The present work compares Industry 4.0 and Society 5.0 in relation to origin, structuring elements and use of technology. The methodology used was a bibliographical research. The result presents a list of 10 characteristics that compare the year of creation of the concept, country of origin, motivating need, level of urgency, focus, scope of application, use of technology, degree of use of artificial intelligence, fundamental elements and developments from the concept. Contributions to the practice are based on the establishment of differences that can contribute so that strategists, investors and production managers can understand the differences and envision a near future.
\end{abstract}

Keywords: Industry 4.0; Society 5.0; Automation; Comparation; Technology.

\section{Resumen}

Las organizaciones han sido introducidas a una nueva forma de organización productiva, denominada Industria 4.0, con la promesa de integrar sus procesos, alta personalización, alta producción y reducción de costos de producción. A partir de los conceptos de Industria 4.0, surgieron varias variaciones, como Logística 4.0, Salud 4.0 y Agricultura 4.0. Sin embargo, fue en Japón donde surgió la idea Society 5.0, que promete la aplicación de todos los aspectos de la cuarta revolución y sus consecuencias en una sociedad totalmente autónoma. El presente trabajo compara Industria 4.0 y Sociedad 5.0 en relación al origen, elementos estructurantes y uso de la tecnología. La metodología utilizada fue una investigación bibliográfica. El resultado presenta un listado de 10 características que comparan año de creación del concepto, país de origen, necesidad motivadora, nivel de urgencia, enfoque, ámbito de aplicación, uso de la tecnología, grado de uso de la inteligencia artificial, elementos fundamentales y desarrollos. del concepto. Las aportaciones a la práctica se basan en el establecimiento de diferencias que pueden contribuir para que estrategas, inversores y gestores de producción puedan comprender las diferencias y vislumbrar un futuro próximo.

Palabras clave: Industria 4.0; Society 5.0; Automatización; Comparación; Tecnología. 


\section{Introdução}

As indústrias estão em busca de aumentar suas eficiências produtivas (Porter, 2005), que corresponde a ter escala em produtividade, aliada a produção de produtos customizados aos clientes (Slack et al., 2015) e desta forma possibilitar o aumento da sua participação no mercado entregando o que cada cliente necessita à um custo reduzido de produção (Kotler, 2012, 2013; Slack et al., 2015).

Os gerentes de produção e a comunidade científica foram apresentados em 2011 na Alemanha (Schwaber, 2018) por uma nova forma de organização produtiva com o emprego da automatização e integralização de seus processos, chamada de quarta revolução industrial ou simplesmente Indústria 4.0 (Ind4), com a promessa de ter alta customização e alta produção em qualquer indústria no mundo (Sacomano et al., 2018).

Em 2016 o Japão apresentou os conceitos da Sociedade 5.0, produto do uso intensivo de tecnologias e inteligência artificial de modo a automatizar, não apenas a indústria, mas o maior número possível de tarefas com o intuito de mitigar a falta de mão de obra que o país apresenta e o envelhecimento da população (GOJ, 2019).

Diante do contexto apresentado, o objetivo desta pesquisa é comparar e determinar as origens, características, bases e a motivação para a criação da Indústria 4.0 e da Sociedade 5.0. Em relação ao espectro da academia científica, o estudo poderá promover a pesquisa dos entrelaçamentos dessas duas abordagens nos aspectos industriais e sociais. Em relação a prática, apresenta as características entre Indústria 4.0 e Sociedade 5.0 que poderá contribuir para que estrategistas, investidores e gestores de produção possam compreender as diferenças e suas similaridades, e que possam vislumbrar um futuro próximo.

\section{Revisão da Literatura}

\subsection{Indústria 4.0}

A Indústria 4.0 necessita da automação de suas linhas de produção e do emprego de tecnologia na integração dos processos (Rübmann et al., 2015; Sacomano et al., 2018; Schwaber, 2018; Zhou et al., 2015). Ela é caracterizada pela inexistência de humanos no processo de produção, no emprego de robôs, Internet of Things (Petroni et al., 2017), sistemas ciber físicos (Sacomano et al., 2018), BigData e Inteligência Artificial (Ford, 2016; Rübmann et al., 2015; Zhou et al., 2015).

O Uso da Inteligência Artificial (IA) na integração dos processos tem sido um grande desafio, desde ao próprio conceito do que vem a ser a inteligência (AI HLEG, 2019; Nilsson, 2009; Stone et al., 2016), como das responsabilidades que ela deve possuir (Stone et al., 2016). Houve algumas tentativas em descrever a IA, como a de uma composição de sistemas de software, e eventualmente do uso de hardware, criados por humanos que atuam na dimensão física ou digital, adquirindo e interpretando dados estruturados ou não estruturados para tomar decisões para alcançar um objetivo complexo (AI HLEG, 2019). Outra definição é que pode ser considerada uma atividade dedicada a fazer as máquinas inteligentes que podem funcionar de forma adequada e previsível em seu ambiente (Nilsson, 2009).

As diversas definições culminaram na criação de um "campo de IA", que estabelece o esforço contínuo para avançar a fronteira da inteligência da máquina de forma contínua e incremental (Stone et al., 2016). Os sistemas de IA podem usar regras simbólicas, aprender um modelo numérico e se adaptar a uma análise do ambiente que está embutido (AI HLEG, 2019).

Independentemente das divergências em sua definição, a IA é um componente importante na Indústria 4.0 para que toda a orquestração dos processos e equipamentos possam coordenar a linha de produção nas indústrias e ser empregada em outras áreas de atuação (Petroni et al., 2017; Sacomano et al., 2018; Schwaber, 2018).

A Indústria 4.0 promoveu o transbordamento de seus conceitos em outras áreas, principalmente na Logística 4.0 (Poli et al., 2018), Saúde 4.0 (Andrade et al., 2019; Badri et al., 2018; Kuziemsky et al., 2019) e Agricultura 4.0 (Monteleone et al., 2019; Zambon et al., 2019). Esses desdobramentos formaram a base para a criação da Sociedade 5.0 (Soc5). 


\subsection{Sociedade 5.0}

A Soc5 é uma evolução (Figura 1) da sociedade que iniciou a partir de grupos de caçadores (Sociedade 1.0), passando pela sociedade agrícola (Sociedade 2.0) com o homem cultivando a terra, do advento da industrialização (Sociedade 3.0) com o surgimento das máquinas a vapor, da geração de novas relações de trabalho e demandando formas de gerenciar a quantidade de informação gerada pela sociedade com o uso de computadores (Sociedade 4.0), culminando no ápice (Sociedade 5.0) da utilização de tecnologias na automação e integralização de processos (GOJ, 2019), e do uso da quarta. revolução tecnológica (Ford, 2016; GOJ, 2019).

Figura 1 - Evolução da Sociedades Baseado em GOJ (2019).

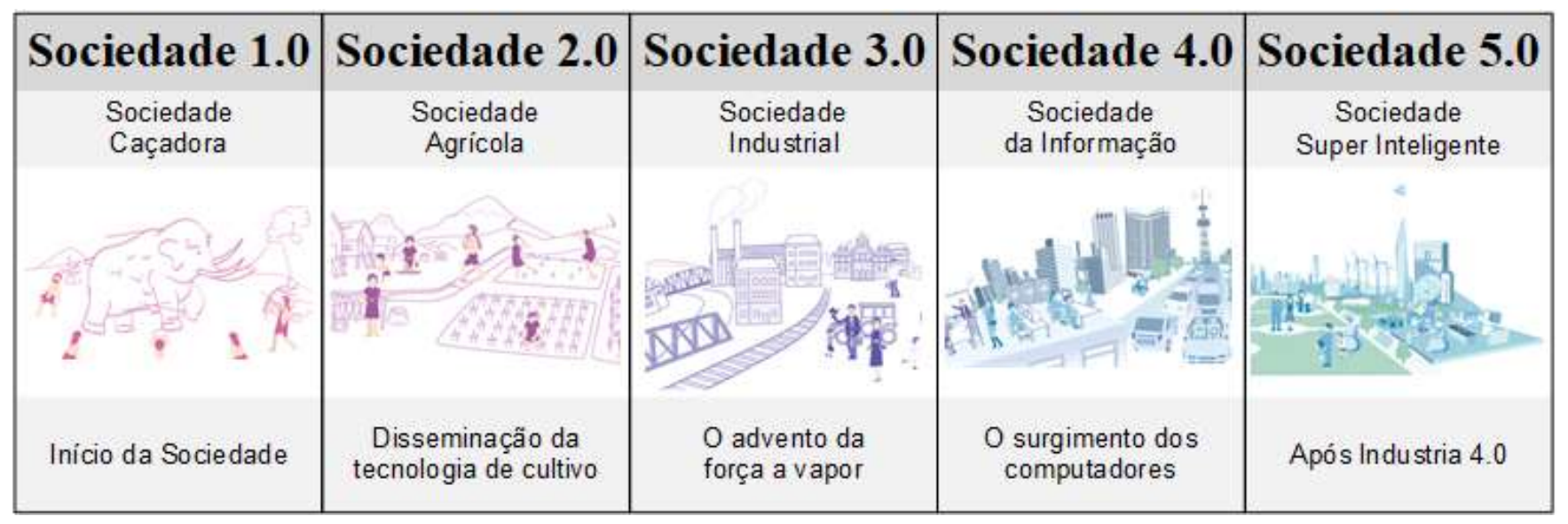

Fonte: Autores.

A Soc5 foi criada pelo Gabinete Oficial do Japão (COJ) em 2016 que buscava criar uma sociedade sustentável para a segurança e o bem-estar dos indivíduos por meio do uso de tecnologias (COJ, 2013) para resolver o problema da população do Japão, que em 10 anos terá a maior parte constituída por idosos, com o emprego de tecnologia para automatizar o maior número de tarefas e consequentemente suprir a falta de mão de obra (CSPH-AI, 2019).

Os elementos estruturantes da Soc5 estão relacionados com a utilização da Indústria 4.0 e seus desdobramentos (Fukuda, 2019), de forma a criar uma integração, que transcende seus conceitos estanques, e possibilitando o funcionamento harmônico como se cada segmento fosse uma engrenagem de um grande relógio, principalmente ao uso da IA (CSPH-AI, 2019; Kuziemsky et al., 2019).

O papel da IA é fundamental para a coordenação das partes, tendo em vista essa importância, o GOJ criou uma série de regras que todas as IA devem seguir para poderem fazer parte da Soc5 e que foi designado como Conselho de Princípios Sociais da IA Humano-Centrica (COJ, 2013; CSPH-AI, 2019).

O Conselho de Princípios Sociais da IA Humano-Centrica realiza reuniões periódicas para estabelecer uma estrutura de conduta esperada para empresas, grupos e sociedade para conscientizar e dignificar o uso da IA (CSPH-AI, 2019), tendo definido os princípios de equidade, responsabilidade e transparência que devem ser seguidos (Shiroishi et al., 2018), como por exemplo as regras de mobilidade para as inteligências artificiais (Glória Júnior \& Reis, 2021).

Existem ao menos três desafios a serem enfrentados pelo governo japonês em relação à implantação do Soc5 (Fukuda, 2019): (1) Mão de obra, a população em idade ativa de 15 a 64 anos tem diminuído desde 2000 e com previsão de ter apenas dois terços da força de trabalho atual; (2) Capital, redução do crédito a empreendedores por aversão ao risco; e (3) Riscos espaciais, em algumas regiões do Japão em 2040 terá 70\% da força de trabalho com mais de 64 anos. 


\section{Metodologia}

O presente trabalho, conforme mostrado na Tabela 1, é de natureza qualitativa (Patton, 2015) e utilizou como metodologia a pesquisa bibliográfica (Martins \& Theóphilo, 2009) com as coletas de dados realizadas em atas de reuniões, frameworks, diretrizes e diversos relatórios oferecidos por documentos digitais disponibilizados pelo escritório do gabinete do governo do Japão e também outras bases de dados digitais.

Tabela 1 - Características da Pesquisa.

\begin{tabular}{lll}
\hline \multicolumn{1}{c}{ Item } & Conteúdo & Autores \\
\hline Natureza & Qualitativa & Patton (2015) \\
\hline Metodologia & Pesquisa Bibliográfica & Martins e Theóphilo (2009) \\
\hline Coleta de Dados & - Atas de reuniões, frameworks, diretrizes e documentos digitais & Comitê Oficial do Japão (2013) \\
& - Outras bases de dados Digitais & Governo Oficial do Japão (2019) \\
\hline
\end{tabular}

Fonte: Autores.

\subsection{Procedimentos Metodológicos}

Os procedimentos realizados (Figura 1) para a construção do artigo foram:

- Etapa 1. Identificar as Características da Indústria 4.0. A consulta aos documentos disponíveis na academia permitiu a identificação das características da Indústria 4.0;

- Etapa 2: Identificar as Características da Sociedade 5.0. A partir dos documentos do Gabinete do Governo do Japão e outras fontes foram identificados os objetivos e características da Sociedade 5.0;

- Etapa 3: Comparar a Indústria 4.0 e a Sociedade 5.0. Elencando as características de cada um dos conceitos foi possível criar as relações dos itens comuns;

- Etapa 4: Apresentar o Resultado da Análise. Foi criada uma tabela com os itens comuns e as características da Indústria 4.0 e da Sociedade 5.0.

Figura 1 - Procedimentos metodológicos.

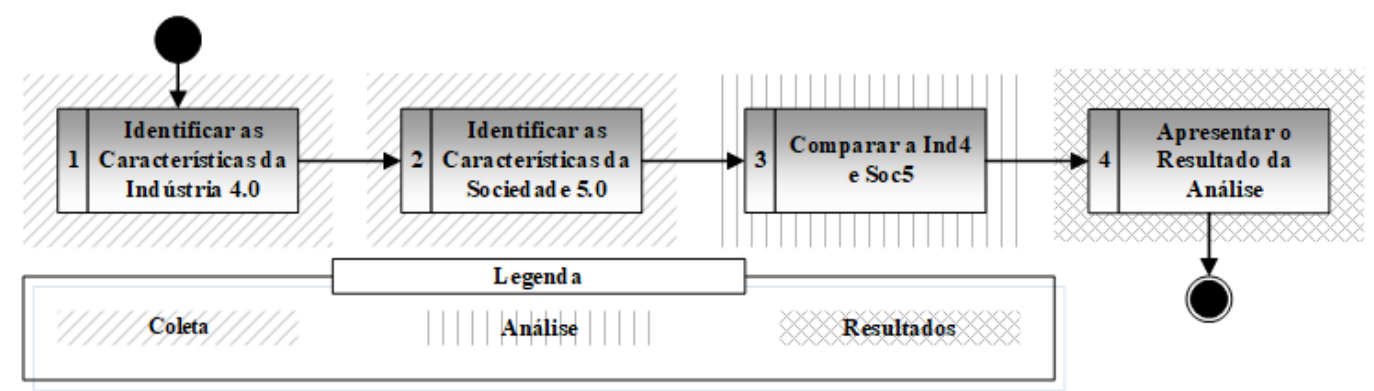

Fonte: Autores.

\section{Resultados e Discussão}

A comparação entre as características dos conceitos da Indústria 4.0 e da Sociedade 5.0 resultaram na Tabela 2 que contém na Coluna 1 os itens a serem comparados e nas Colunas 2 e 3 os resultados das análises da Ind4 e Soc5 respectivamente. Essa sequência foi estabelecida devido a ordem cronológica de suas criações. 
Os termos tiveram como ano de criação (C01) e o país de origem (C02) identificados na literatura científica como sendo a Ind4 cunhada em 2011 na Alemanha (Schwaber, 2018) e a Sociedade 5.0 foi estabelecida em 2016 no Japão pelo Gabinete do Governo Japonês (GOJ, 2019).

Outro ponto analisado é em relação a motivação da criação dos conceitos (C03) em que na Ind4 é uma proposição de otimização da produção nas indústrias (Sacomano et al., 2018; Schwaber, 2018) e a Soc5 é proveniente da necessidade de mão de obra tendo em vista que o envelhecimento da população japonesa (GOJ, 2019).

O nível de urgência (C04) requerida no desenvolvimento da Ind4 possui caráter de melhoria de processo de produção em diversos setores, desta forma, sem uma datação ou outro elemento relevante que determine uma datação (Rübmann et al., 2015; Sacomano et al., 2018; Zhou et al., 2015). A Soc5 possui previsão de colapso de mão de obra até 2040, diante disso, deve ser implementada em caráter de urgência o mais breve possível (Fukuda, 2019; GOJ, 2019; Shiroishi et al., 2018)

Há divergência em relação ao foco (C05) e o escopo (C06) de onde deve ser aplicado os conceitos, enquanto a Ind4 é direcionada a melhoria da produção nas indústrias em nível mundial (Schwaber, 2018), a Soc5 é destinada a melhoria e sobrevivência de um país específico, no caso o Japão (COG, 2019; COJ, 2013).

O emprego da tecnologia (C07) possui como característica na Ind4 o uso da automação e os elementos estruturantes com emprego de alta tecnologia, como IoT e Bigdata (Sacomano et al., 2018; Schwaber, 2018). Em relação a Soc5, além desses itens, existe preocupação e maior emprego de tecnologia, sobretudo em relação a IA (GOJ, 2019).

O uso da IA (C08) é predominante na Ind4 e em seus desdobramentos para poder haver a integração de suas partes (Schwaber, 2018), mas na Soc5 essa interação vai muito além, tornando o uso da IA um elemento dominante, sendo primordial para controlar todas as nuances da sociedade e suas interrelações (CSPH-AI, 2019).

Os elementos fundamentais (C09) para a criação da Ind4 é a automação das linhas de produção que serão integradas pelos processos (Rübmann et al., 2015; Sacomano et al., 2018; Schwaber, 2018; Zhou et al., 2015). A Soc5 possui como alicerces a Ind4 e todas as suas vertentes (Fukuda, 2019; GOJ, 2019; Shiroishi et al., 2018).

Os desdobramentos (C10) que foram identificados constam apenas na Ind4, tendo como principais a Logística 4.0 (Poli et al., 2018), Saúde 4.0 (Andrade et al., 2019; Badri et al., 2018; Kuziemsky et al., 2019) e Agricultura 4.0 (Monteleone et al., 2019; Zambon et al., 2019).

Ao iniciar a comparação entre Ind4 e Soc5 o motivo que culminou na criação dos conceitos é um ponto de inflexão e que determina todos os outros itens: a Ind4 possui como objetivo a melhoria da produção e integração dos processos e não visa solucionar uma situação de vida ou morte, mas de conseguir mais lucros para as indústrias de qualquer país, o que o torna atraente para as empresas e pesquisadores em todo o mundo.

Diferentemente da Ind4, a Soc5 foi criada a partir de um problema presente em um país específico, no caso o Japão, que está diante de um desafio social relevante e urgente que é o envelhecimento da sua população. As constantes reuniões do conselho do Gabinete do Japão e o investimento monetário são indícios da seriedade daquele país em busca de uma solução viável até 2040. 
Tabela 2 - Comparações entre Indústria 4.0 e Sociedade 5.0.

\begin{tabular}{lccc}
\hline$\#$ & Item & Indústria 4.0 & Sociedade 5.0 \\
\hline C01 & Ano de Criação do Conceito & 2011 & 2016 \\
\hline C02 & País de Origem & Alemanha & Japão \\
\hline C03 & Necessidade Motivadora & Otimização & Envelhecimento \\
\hline C04 & Nível de Urgência & Normal & Emergencial \\
\hline C05 & Foco & Indústria & País \\
\hline C06 & Escopo de Aplicação & Alto & Alto/Altíssimo \\
\hline C07 & Emprego de Tecnologia & Dominante \\
\hline C08 & Uso de IA & Predominante & Indústria 4.0 \\
\hline C09 & Elementos Fundamentais & Log4/Agro4/Hlt4 & Nenhum \\
\hline C10 & Desdobramentos & & \\
\hline
\end{tabular}

Fonte: Autores.

A Soc5 também possui um problema de implantação de sua sociedade, pois sua base está relacionada com o desenvolvimento das vertentes da quarta revolução industrial para poder estabelecer um mínimo de integração, desta forma, cria-se uma dependência tecnológica e de processos que o Japão pode não ter tempo hábil para esperar.

É possível conjecturar que a Soc5 pode encontrar dificuldades em ser desenvolvida devido a sua amplitude de atuação, pois visa exclusivamente a sociedade japonesa (CSPH-AI, 2019; GOJ, 2019). É possível encontrar no Conselho de Princípios Sociais da IA Humano-Centrica premissas que são aplicadas somente ao povo daquele país, como a forma de educação, contato com a tecnologia e costumes (CSPH-AI, 2019), tornando as pesquisas restritas somente a esse objeto de estudo. Diferentemente da aplicação da Ind4 que é abrangente e diversificada, em que é possível utilizar diferentes objetos de estudos em múltiplos países, e assim, possibilitando que mais pesquisadores realizem estudos sobre o tema.

Outro ponto observado é o fato que a Ind4 possui vertentes, como a Logística 4.0 e a Saúde 4.0, mas a Soc5 não possui. Isto pode ser explicado devido à complexidade inerente da Soc5, pois inclui todos os aspectos de uma sociedade e não apenas uma linha de produção ou a organização de um galpão.

A pesquisa e desenvolvimento em tecnologias de vanguarda requere investimentos financeiros (Glória Júnior et al., 2017) e a situação do Japão apresenta falta de capital para operações de risco (Fukuda, 2019) o que pode comprometer a implantação da Soc5. Esse cenário poderia mudar caso as premissas fossem alteradas para poderem ser implantadas em qualquer outro país.

\section{Considerações Finais}

As indústrias foram apresentadas na Alemanha em 2011 à quarta revolução industrial, denominada Indústria 4.0, e que possui como características a integralização dos processos, inexistência de humanos na produção e o emprego de tecnologia no processo de automatização, com o uso de robôs, Internet of Things, sistemas ciber-físico, Bigdata e da Inteligência Artificial. A Indústria 4.0 provocou vários desdobramentos na logística, saúde e agricultura. A união da Indústria 4.0 e suas vertentes formam a base da Sociedade 5.0, termo criado em 2016 no Japão, para promover a automatização e integração de sua sociedade oriunda da falta de mão de obra devido ao envelhecimento de sua população.

A pesquisa realizou a comparação de 10 itens entre a Indústria 4.0 e a Sociedade 5.0 e como resultado obteve que o termo Indústria 4.0 foi cunhado na Alemanha em 2011 com a promessa de otimização da produção em qualquer país e com o emprego de tecnologias, como a Inteligência Artificial, a sua urgência estava ligada ao lucro e não a vidas humanas, está baseada na automação e gerou desdobramentos como a Logística 4.0, Agricultura 4.0 e Saúde 4.0. 
Em relação a Sociedade 5.0 surgiu no Japão em 2016 com a necessidade de gerar processos automatizados no país de origem do termo e com o emprego de alta tecnologias, dominada pelo emprego da Inteligência Artificial. A urgência de sua aplicação está ligada ao envelhecimento dos japoneses. Possui seus alicerces na Indústria 4.0 e suas nuances. Ela não apresenta até o momento algum desdobramento.

As contribuições para a academia científica são a de apresentar e promover estudos de Indústria 4.0 e Sociedade 5.0 em relação aos seus aspectos e suas interrelações. As contribuições para a prática são do estabelecimento das diferenciações que podem contribuir para que estrategistas, investidores e gestores de produção possam compreender as diferenças e vislumbrarem um futuro próximo. Futuros trabalhos incluem a comparação e contribuição dos desdobramentos da Indústria 4.0 para a Sociedade 5.0.

\section{Agradecimentos}

O pós-doutorando Irapuan Glória Júnior agradece ao Programa de Pós-Graduação Stricto Sensu em Engenharia de Produção (PPGEP) da Universidade Paulista (UNIP).

\section{Referências}

AI HLEG. (2019). High-Level Expert Group on Artificial Intelligence: Ethics Guidelines For Trustworthy AI. 1-41.

Andrade, N., Costa Neto, P. L. O., Torres, J. G. M., Glória Júnior, I., Scheidt, C. G., \& Gazel, W. (2019). E-Health: A framework proposal for interoperability and health data sharing. A Brazilian Case. IFIP International Conference on Advances in Production Management Systems, 1, $544-551$.

Badri, A., Boudreau-Trudel, B., \& Souissi, A. S. (2018). Occupational health and safety in the industry 4.0 era: A cause for major concern? Safety Science, 109, 403-411. https://doi.org/10.1016/j.ssci.2018.06.012

COG. (2019). Cabinet Office Government of Japan: Automatic Driving. https://www.gov-online.go.jp/cam/s5/eng/\#sceneModal14

COJ. (2013). Cabinet Office of Japan: Social Principles of Human-Centric AI. 1-15.

CSPH-AI. (2019). Council for Social Principles of Human-centric AI: Social Principles of Human-Centric AI. https://www8.cao.go.jp/cstp /english/humancentricai.pdf

Ford, M. (2016). Industry 4.0: Making the First Move. Surface Mount Technology, 31(7), 30-36.

Fukuda, K. (2019). Science, technology and innovation ecosystem transformation toward society 5.0. International Journal of Production Economics.

Glória Júnior, I., Moraes, M. O., Araújo, M. B., \& Torres, J. G. M. (2017). Engenharia da Produção—Tecnologia \& Informação—Vol.2. PerSe.

Glória Júnior, I., \& Reis, J. G. M. dos. (2021). Logística 4.0 e Inteligência Artificial na Sociedade 5.0. 1-13.

GOJ. (2019). Society 5.0 [Government of Japan]. Government of Japan - Public Relations Office - Society 5.0. https://www.govonline.go.jp/cam/s5/eng/\#sceneModal13

Kotler, P. (2012). Marketing 3.0. As forças que estão definindo o novo marketing centrado no ser humano. Elsevier.

Kotler, P. (2013). Administração de Marketing. Pearson.

Kuziemsky, C., Maeder, A. J., John, O., Gogia, S. B., Basu, A., Meher, S., \& Ito, M. (2019). Role of Artificial Intelligence within the Telehealth Domain: Official 2019 Yearbook Contribution by the members of IMIA Telehealth Working Group. 28, 35-40. https://doi.org/10.1055/s-0039-1677897

Martins, G. de A., \& Theóphilo, C. R. (2009). Metodologia da Investigação Científica Para Ciências Sociais Aplicadas (2a ed). Atlas.

Monteleone, S., de Moraes, E. A., \& Maia, R. F. (2019). Analysis of the variables that affect the intention to adopt Precision Agriculture for smart watermanagement in Agriculture 4.0 context. 2019 Global IoT Summit (GIoTS), 1-6.

Nilsson, N. J. (2009). The quest for artificial intelligence. Cambridge University Press.

Patton, M. (2015). Qualitative Research \& Evaluation Methods: Integrating Theory and Practice (4a ed). SAGE Publications.

Petroni, B. C. A., Glória Júnior, I., \& Gonçalves, R. F. (2017). Impacto da Internet das Coisas na indústria 4.0: Uma Revisão Sistemática da Literatura. 1-6.

Poli, G. A., Saviani, T. N., \& Glória Júnior, I. (2018). Logistica 4.0: Uma Revisão Sistemática. 1, 100-110.

Porter, M. E. (2005). Estratégia Competitiva_Técnicas Para Análise de Indústrias e da Concorrência. GEN Atlas. 
Research, Society and Development, v. 10, n. 11, e23101119192, 2021

(CC BY 4.0) | ISSN 2525-3409 | DOI: http://dx.doi.org/10.33448/rsd-v10i11.19192

Rübmann, M., Lorenz, M., Gerbert, P., Waldner, M., Justus, J., Engel, P., \& Harnisch, M. (2015). Industry 4.0: The future of productivity and growth in manufacturing industries. http://www.inovasyon.org/pdf/bcg.perspectives_Industry.4.0_2015.pdf

Sacomano, J. B., Gonçalves, R. F., Bonilla, S. H., da Silva, M. T., \& Sátyro, W. C. (2018). Indústria 4.0. Editora Blucher.

Schwaber, K. (2018). A quarta revolução industrial. Edipro.

Shiroishi, Y., Uchiyama, K., \& Suzuki, N. (2018). Society 5.0: For human security and well-being. Computer, 51(7), 91-95.

Slack, N., Chambers, S., \& Johnston, R. (2015). Administração da produção (Vol. 4). Atlas.

Stone, P., Brooks, R., Brynjolfsson, E., Calo, R., Etzioni, O., Hager, G., Hirschberg, J., Kalyanakrishnan, S., Kamar, E., Kraus, S., Leyton-Brown, K., Parkes, D., Press, W., Saxenian, A., Shah, J., Tambe, M., \& Teller, A. (2016). Artificial Intelligence and Life 2030. One Hundred Year Study on Artificial Intelligence: Report of the 2015-2016 Study Panel. 52.

Zambon, I., Cecchini, M., Egidi, G., Saporito, M. G., \& Colantoni, A. (2019). Revolution 4.0: Industry vs. Agriculture in a future development for SMEs. Processes, 7(1), 1-36.

Zhou, K., Liu, T., \& Zhou, L. (2015). Industry 4.0: Towards future industrial opportunities and challenges. 2147-2152. 